International Conference on Metallurgical Coatings and Thin Films

San Diego, CA, April 22-26, 2002

BNL -69063

\title{
METALLIZATION OF CERAMIC VACUUM CHAMBERS FOR SNS RING INJECTION KICKER MAGNETS**
}

\author{
P. He, H.C. Hseuh, R.J. Todd \\ Collider-Accelerator Department \\ Brookhaven National Laboratory \\ Upton, NY 11973
}

Abstract

Ceramic chambers will be used in the pulsed kicker magnets for the injection of $\mathrm{H}^{-}$ into the US Spallation Neutron Source (SNS) accumulator ring. There are two reasons for using ceramic chambers in kickers: (1) to avoid shielding of a fast-changing external magnetic field by metallic chamber walls; and (2) to reduce heating due to eddy currents. The inner surfaces of the ceramic chambers will be coated with a conductive layer, possibly titanium $(\mathrm{Ti})$ or copper $(\mathrm{Cu})$ with a titanium nitride(TiN) overlayer, to reduce the beam coupling impedance and provide passage for beam image current. This paper describes the development of sputtering method for the $0.83 \mathrm{~m}$ long $16 \mathrm{~cm}$ inner diameter ceramic chambers. Coatings of $\mathrm{Ti}, \mathrm{Cu}$ and $\mathrm{TiN}$ with thicknesses up to $10 \mu \mathrm{m}$ were produced by means of DC magnetron sputtering. The difficulty of coating insulators was overcome with the introduction of an anode screen. Films with good adhesion, uniform longitudinal thickness, and conductivity were produced.

\footnotetext{
${ }^{*}$ Work performed under the auspices of the U.S. Department of Energy.
} 


\section{INTRODUCTION}

Brookhaven is undertaking the design, construction and commissioning of the SNS accumulator ring. The SNS injection system is composed of conventional window-frame ferrite-core kicker magnets. The ferrite yokes are mounted around ceramic vacuum chambers metallized on the inside surface. Ceramic chambers are used to allow the external time-varying field to penetrate the vacuum chamber, while a thin metallic coating is required to carry beam-induced image currents. The coating material and thickness are chosen based on the following requirements[1]:

(i) external fields must penetrate the coating

(ii) the rise time of the external field must be preserved

(iii) electrical continuity to the adjoining vacuum chambers must be provided

(iv) the film must tolerate magnet pulsing (remain adherent, no arcing, etc)

(v) subject to ohmic heating from the beam image current andeddy current heating from magnetic pulsing

(vi) low secondary electron yield (SEY)

The ceramic chambers, totaling six for the eight injection kicker magnets, have a $16 \mathrm{~cm}(\mathrm{ID})$ and are $0.83 \mathrm{~m}$ long. Alumina $\left(\mathrm{Al}_{2} \mathrm{O}_{3}\right)$ ceramic was chosen as vacuum chamber because of its greater mechanical strength, excellent UHV properties and ease of brazing to metal flanges. The coating must have a minimum conductivity to avoid attenuation and deformation of the magnetic field. A homogenous and well adherent layer is required to avoid destructive sparks due to the high voltage pulse induced by the pulsed magnetic field. Either $\mathrm{Ti}$ or $\mathrm{Cu}$ with a TiN overlayer (to reduce SEY), have been chosen as the potential coating materials for their high melting point, high sputtering efficiency, low 
resistivity and reactivity, resulting in good adhesion to the alumina. This paper presents the development of the coating process and the properties of these coatings.

\section{COATING METHOD}

Magnetron sputtering is currently being used to coat the SNS half-cell chambers with TiN.[2] For this application, a cathode was developed which produced TiN films of good uniformity and composition. Commercially available Alnico magnets 1_" diameter and 2" in length were inserted in a 1 _" diameter tubing (grade-2 $\mathrm{Ti}$ or $\mathrm{OFHC} \mathrm{Cu}$ ). A _" hole in the center of the magnets allowed for water cooling of the cathode. The deflection of the long cathode was found to be within acceptable limits. The magnets were stacked with opposing poles using non-ferrous _" spacers resulted in a looping magnetic field of sufficient strength to project from the cathode surface. This "low cost" cathode worked in conjunction with a $10 \mathrm{KW}$ DC power supply to produce the satisfactory field $(0.08 \mathrm{~T} \sim 0.1 \mathrm{~T})$ and discharge plasma as shown in Figure 1.

The theoretical uniformity of sputtered films was calculated by treating each plasma ring as a point source. The thickness distribution on a flat surface (i.e. chamber wall) located $8.3 \mathrm{~cm}$ away was calculated using a point source cosine distribution [3]. Based on a plasma ring spacing of $6.6 \mathrm{~cm}$, the superposition of the individual thickness distributions resulted in an overall uniformity within $010 \%$ (Figure 2). This cathode design was used to coat $\mathrm{Cu}$, Ti or TiN. The coating development was done in a $20 \mathrm{~cm}$ diameter test chamber (Figure 3) allowing insertion of either glass or ceramic tubes.

\section{COATING DEVELOPMENT}

\section{TiN coating on ceramic tube}

The first phase of the development involved coating of a bare ceramic pipe with 6 $\mu \mathrm{m}$ of TiN. The $16 \mathrm{~cm} \mathrm{ID} 83 \mathrm{~cm}$ long ceramic pipe was positioned at the midpoint of the test chamber. The chamber was evacuated and baked using external heating tapes to 250 
${ }^{\circ} \mathrm{C}$ for 48 hours to remove water vapor and other surface contaminants. The chamber was maintained at $250^{\circ} \mathrm{C}$ during the sputtering process to minimize oxide formation on the substrate. Following residual gas analysis to ensure the absence of contaminants, Ar and $\mathrm{N}_{2}$ with purity better than $99.9999 \%$ were fed into the test chamber. The flow rates were adjusted using the mass flow controllers and were $13.5 \mathrm{sccm}$ for Ar and $7.5 \mathrm{sccm}$ for N2. Total pressure in the chamber was $\sim 5$ mTorr. The discharge was current limited at $10 \mathrm{~A}$. The discharge voltage was $340 \mathrm{VDC}$. The deposition rate was $\sim 0.07 \mathrm{~nm} / \mathrm{sec}$ (TiN) as measured by a quartz crystal thickness monitor.

The reactive process was controlled using partial pressure feedback of nitrogen gas[4]. Mass flow controllers were used to introduce both argon and nitrogen, while a high pressure residual gas analyzer was used to monitor nitrogen partial pressure. The nitrogen was introduced through a _" diameter Ti tube which runs the length of the cathode. Small (i.e. $\sim 10^{-2}$ inch dia.) holes located every six inches provided even distribution of reactive gas, which was necessary for consistent color and composition throughout the length of the chamber. A hysteresis curve of nitrogen consumption was generated (Figure 4). It was found that the best results were obtained by first nitriding the cathode by increasing $\mathrm{N}_{2}$ flow above point $B$ and then lowering to just above point $D$. By operating in this condition, a stable plasma was formed, resulting in a uniform longitudinal color.

Difficulties with coating insulators

Initial coatings of TiN on ceramic revealed less than ideal thickness uniformity along the length of the tube. The resistance of the film, which is proportional to the coating thickness was measured using a four-point probe. The thickness distribution of the film along the length of the ceramic tube ( $\mathrm{z}$ direction) was derived using a resistivity value of $45 \mu \Omega . c m$ for TiN. The distribution, as shown in Figure 5, was not linear. It was suspected that a charge buildup on the ceramic had distorted the electrical field and 
inhibited the discharge within the tube. The observed coating was a result of the discharge beyond the ends of the ceramic tube.

To verify this hypothesis, a second run was made using two glass tubes, which were more readily available than the ceramic tubes. The use of glass tubes also enabled viewing of the discharge pattern through viewports located along the test chamber. One tube had two, well grounded 1" wide Aluminum strips running along the inner wall of the glass tube acting as an anode trap. The second tube was installed without these grounding strips. With the discharge initiated, there was a familiar, well formed, discharge within the tube with the anode trap, but no visible discharge in the second.

The longitudinal resistance was again measured using the four-point probe. The thickness distribution was derived using a thin film resistivity of about $95 \mu \Omega . c m$ for Ti As shown in Figure 6, the thickness distribution of the glass tube without $\mathrm{Al}$ grounding stripes, is very similar to that of the ceramic tube. The glass tube with Al strips has much better longitudinal thickness uniformity. This verified our hypothesis that the electric field built up inside the insulating tube, affects the discharge, and consequently the thickness uniformity.

\section{Copper on glass pipes}

A 1.5" diameter OFHC copper tube was used as the cathode in this test. A grounded anode screen, ensuring the uniform distribution of the DC field and enabling the conduction of electrical charges, was made using 304 stainless steel mesh (Figure 7).

Copper was sputtered in two tests, onto both smooth and etched glass tubes measuring $17 \mathrm{~cm}$ in diameter and $91 \mathrm{~cm}$ in length. The $10 \mathrm{~cm}$ diameter anode screen was positioned concentric with the $\mathrm{Cu}$ cathode and glass tube. A $90 \mathrm{~cm}$ magnet strings was centered longitudinally in the glass tube. The discharge was current limited at 5 amps with 
a discharge voltage of $400 \mathrm{VDC}$. The deposition rate was $20 \mathrm{~nm} / \mathrm{s}$. The thickness distribution of the copper film on the glass tubes was found to be within $20 \%$ end to end.

\section{FILM PROPERTIES}

\section{TiN Composition}

Stainless steel witness coupons were mounted in the bottom ports along the test chamber. Film stoichiometry and thickness of the coupons were analyzed by Auger electron spectroscopy (AES). The AES system used is a modified PHI4300 Scanning Auger Microprobe (SAM) with incident electron beam of $10 \mathrm{keV}$ and $10 \mathrm{nA}$. Survey scans for all elements ( $\mathrm{Li}$ to $\mathrm{U}$ ) were acquired on the as-received surfaces. Depth profiles were obtained by alternating an acquisition cycle with a sputtering cycle. The sputtering cycles remove material from sample surface using a $4 \mathrm{keV} \mathrm{Ar}^{+}$source rastered over a $3 \mathrm{~mm} \times 3 \mathrm{~mm}$ area. To eliminate crater-wall effect, the data were acquired from a smaller region in the center of the sputtered area. Typical results of AES analysis are shown in Figure 8.

\section{Adhesion}

The coating adhesion to the substrate surface is one of the most important properties. Two methods have been used for adhesion test. The first one is the simple "scotch-tape test" in which adhesive tape is attached to the film and pulled quickly in an attempt to remove the film. The second more demanding test requires scribing a grid of finely spaced cuts through the film down to the substrate, typically a $4 \mathrm{~mm} \times 4 \mathrm{~mm}$ grid. This portion of the film is then subjected to the tape test. This is a more stringent test to determine the physical bonding to the substrate and was also used for our films. Both of these methods were applied to the ceramic tube and the glass tubes. The results show good adhesion to both the ceramic tube and etched glass tube and poor adhesion to the smooth glass. It was also observed that adhesion is reduced as thickness increases. 


\section{SUMMARY}

DC magnetron sputtering has been developed to coat the SNS ring injection kicker ceramic vacuum chamber with $\mathrm{TiN}, \mathrm{Ti}$ and $\mathrm{Cu}$. The difficulties in producing a film with uniform thickness within a long insulating chamber have been overcome with the development of a unique cathode with internal magnet string and an anode screen.

\section{ACKNOWLEDGEMENTS}

The authors would like to thank R. Sabatini of BNL for AES analysis of some coated samples and the C-A Vacuum Group personnel in setting up the experiment.

\section{REFERENCES}

[1] A.W. Chao and M. Tigner, 'Handbook of Accelerator Physics and Engineering', World Scientific, Singapore, 1999.

[2] P.He, H.C. Hseuh, M. Mapes, R. Todd and D. Weiss, Proc. of 2001 Particle Accel. Conf., Chicago, USA, (2001), in press.

[3] J.C. Helmer and C.E. Wickersham, J. Vac. Sci. Technol. A4, 408 (1986).

[4] W.D. Sproul, P.J. Rudnik and M.E. Graham, Surface and Coating Technol., 39/40, 355 (1989). 


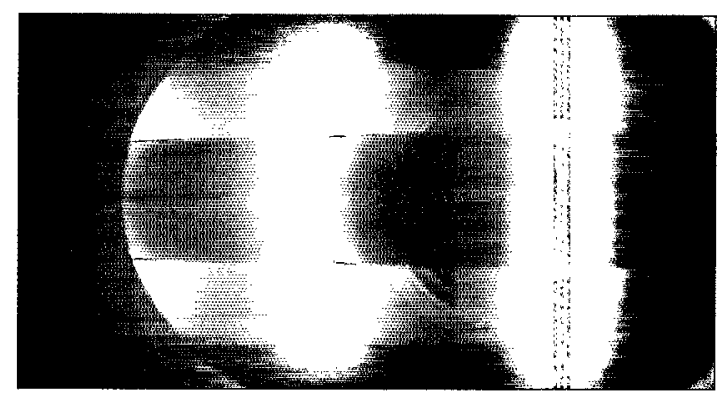

Figure 1. Discharge characteristics of DC magnetron with bright rings at the locations of the magnets.

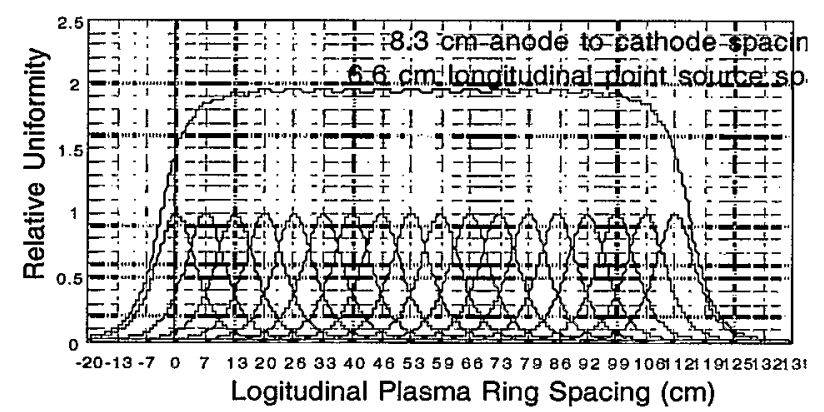

Figure 2. Calculated longitudinal film uniformity based on point source cosine distribution. 


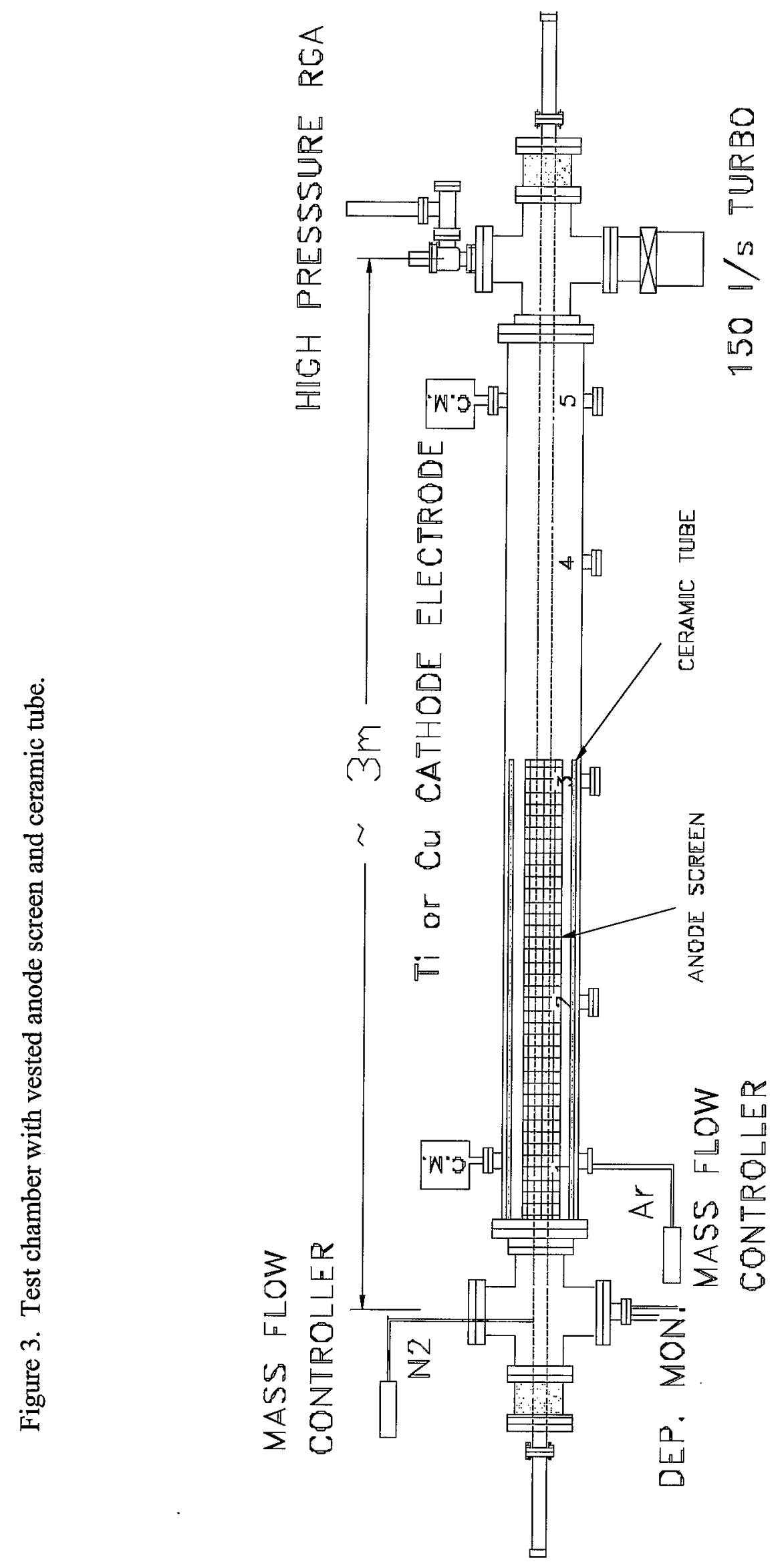


Figure 4. Nitrogen Hysteresis, Constant Ar Flow

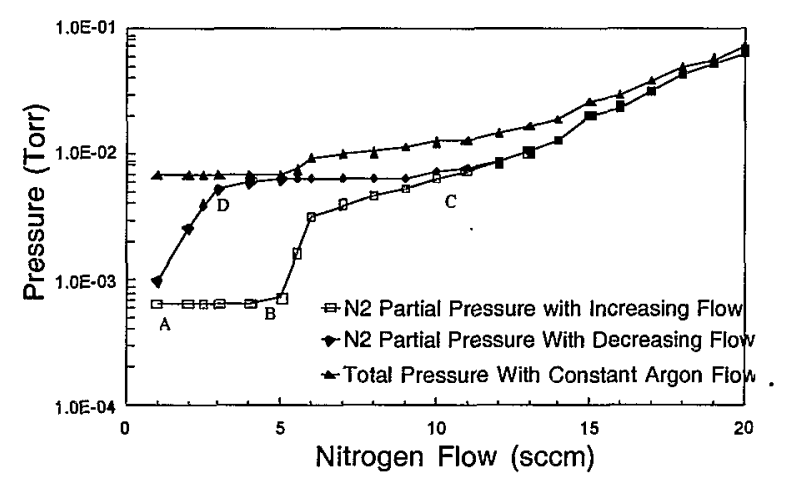

TiN thickness(um)

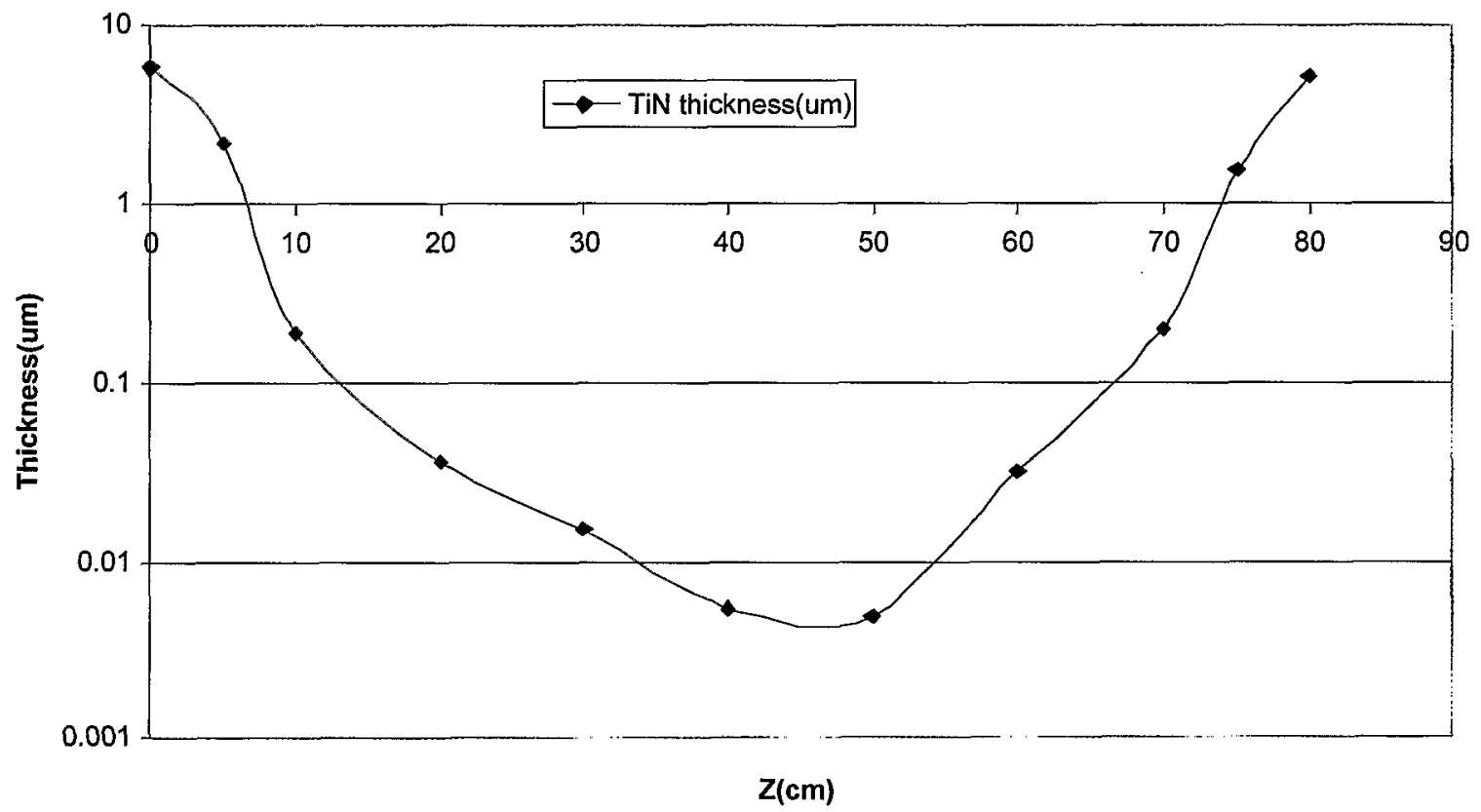

Figure 5. Thickness variation as a result of the absence of discharge within the ceramic tube. 
Thickness Distribution

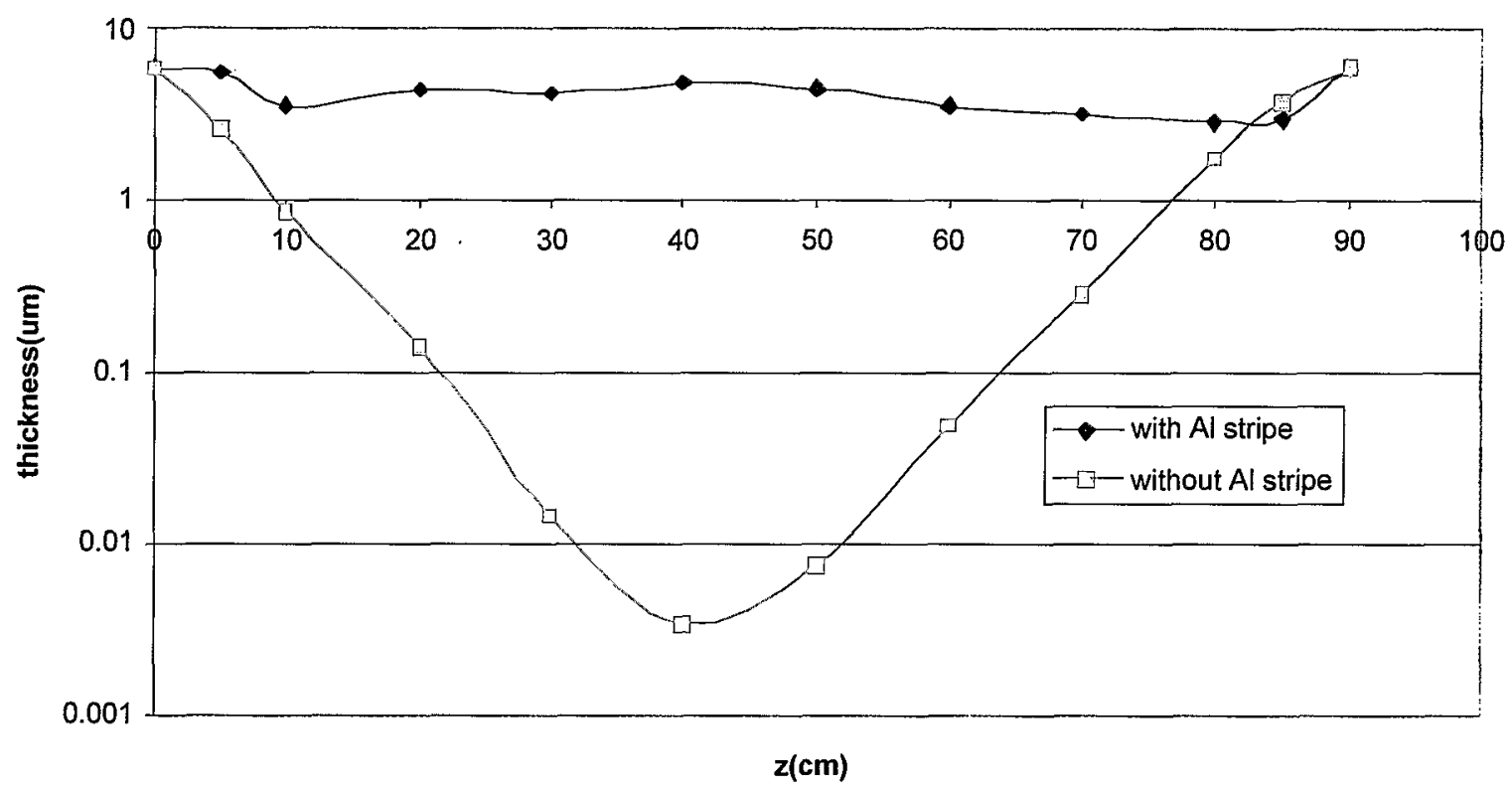

Figure 6. Thickness distribution of Ti on glass tubes with and without the presence of anode strips within the tube. 


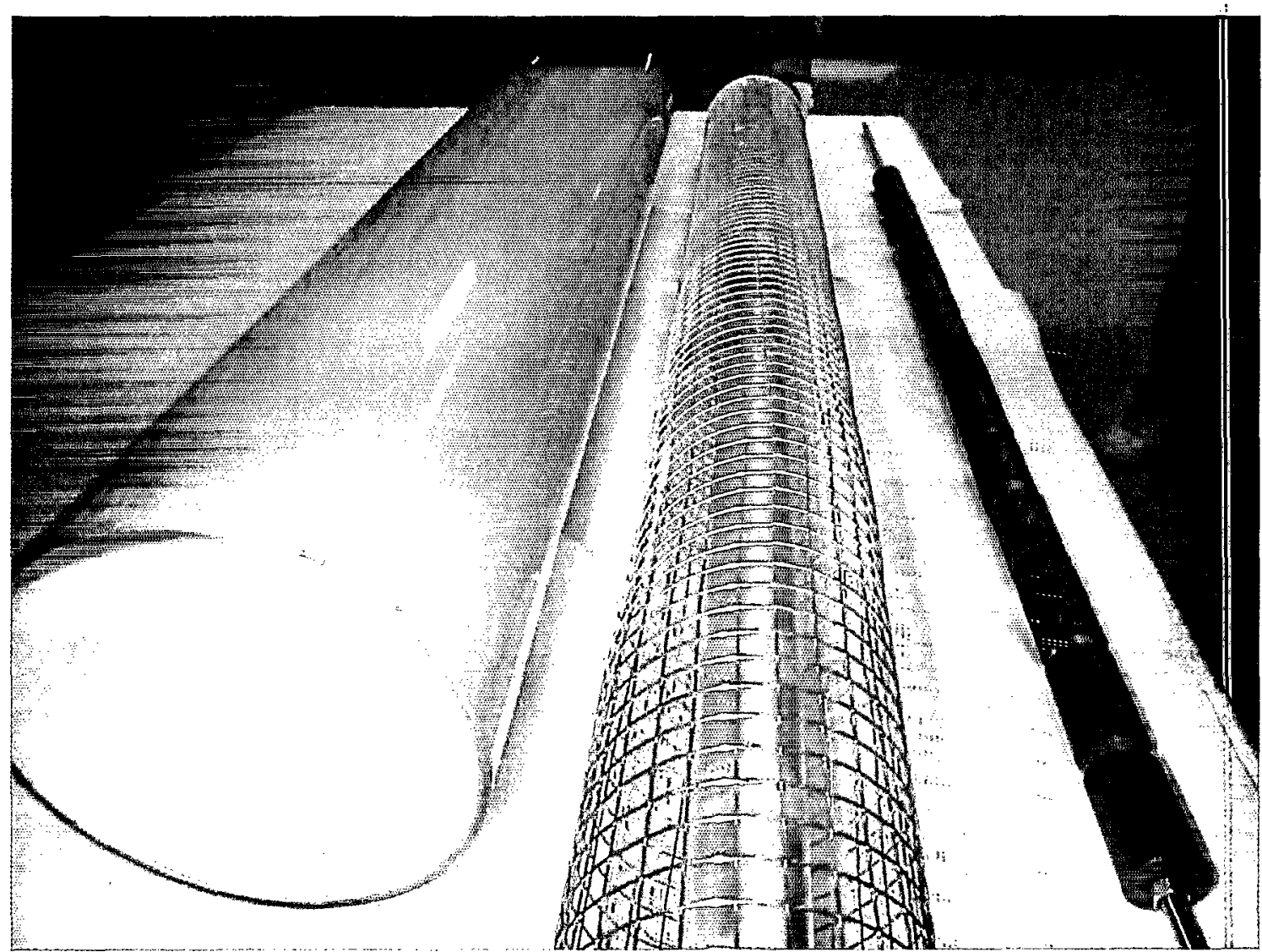

Figure 7. Anode screen shown with $\mathrm{Cu}$ cathode, magnet string and coated glass tube.

AEB Survey spoetres

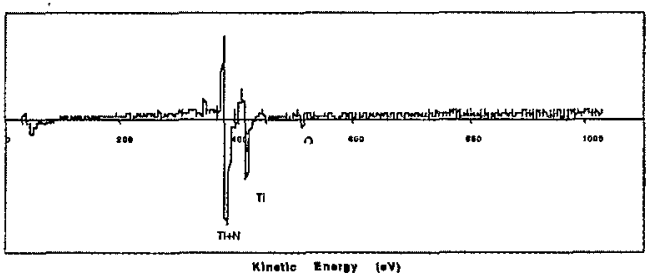

Figure 8. AES analysis of TiN sample, showing little oxygen or carbon contamination and correct stoichiometric ratio of $\mathrm{Ti}$ to $\mathrm{N}$. 